\title{
Confirmation of associations between ion channel gene SNPs and QTc interval duration in healthy subjects
}

\author{
L Gouas $^{*, 1,2}$, V Nicaud ${ }^{3}$, S Chaouch ${ }^{1,2}$, M Berthet ${ }^{1,2}$, A Forhan ${ }^{4}$, J Tichet ${ }^{5}$, L Tiret ${ }^{3}$, B Balkau ${ }^{4}$, \\ P Guicheney ${ }^{1,2,6}$ and The D.E.S.I.R Study Group
}

\begin{abstract}
${ }^{1}$ INSERM, U582, Institut de Myologie, Paris, France; ${ }^{2}$ Université Pierre et Marie Curie-Paris 6, UMR S582, IFR14, Paris, France; ${ }^{3}$ INSERM, UMRS 525, Université Pierre et Marie Curie-Paris 6, Paris, France; ${ }^{4}$ INSERM U780, IFR 69, Villejuif, France; ${ }^{5}$ IRSA, La Riche, France; ${ }^{6}$ AP-HP, Groupe Hospitalier Pitié-Salpêtrière, Service de Biochimie, Paris, France
\end{abstract}

Population-based association studies have identified several polymorphic variants in genes encoding ion channel subunits associated with the electrocardiographic heart-rate-corrected QT (QTC) length in healthy populations of Caucasian origin (KCNH2 rs1805123 (K897T) and rs3815459, SCN5A rs1805126 (D1819D), 1141-3 C >A, rs1805124 (H558R), and IVS24+116 G >A, KCNQ1 rs757092, KCNE1 IVS2-128 G $>$ A and rs1805127 (G38S), and KCNE2 rs2234916 (T8A)). However, few of these results have been replicated in independent populations. We tested the association of SNPs KCNQ1 rs757092, KCNH2 rs3815459, SCN5A IVS24 + 116 G $>$ A, KCNE1 IVS2-128 G > A and KCNE2 rs2234916 with QTc length in two groups of 200 subjects presenting the shortest and the longest QTc from a cohort of 2008 healthy subjects. All polymorphisms were in Hardy-Weinberg equilibrium in both groups. The minor allele SCN5A IVS24+116 A was more frequent in the group of subjects with the shortest QTc, whereas the minor alleles KCNQ1 rs757092 $\mathrm{G}$ and $K C N H 2$ rs3815459 A were more frequent in the group with the longest QTc. There was no significant difference for KCNE1 IVS2-128 G > A and KCNE2 rs2234916 between the two groups. Haplotype analysis showed a twofold increased risk of QTc lengthening for carriers of the haplotype, combining alleles $C$ and A of the two common KCNE1 SNPs, IVS2-129 C>T (rs2236609) and rs1805127 (G38S), respectively. In conclusion, our study confirms the reported associations between QTc length and KCNQ1 rs757092 and KCNH2 rs3815459.

European Journal of Human Genetics (2007) 15, 974-979; doi:10.1038/sj.ejhg.5201866; published online 30 May 2007

Keywords: gene polymorphisms; ion channels; QT interval

\section{Introduction}

The cardiac ventricular repolarization process, which is measured by the QT interval length on the electrocardiogram (ECG), is known to be influenced by various

*Correspondence: $\operatorname{Dr}$ L Gouas, Service de Cytogénétique Médicale, Faculté de Médecine et de Pharmacie, 28 place Henri Dunant, 63001 Clermont-Ferrand Cedex, France.

Tel: + 334731781 00; Fax: + 334732691 82;

E-mail: Igouas@chu-clermontferrand.fr

Received 30 November 2006; revised 26 April 2007; accepted 2 May 2007; published online 30 May 2007 parameters (heart rate, ${ }^{1}$ age, ${ }^{2}$ sex ${ }^{3}$ and medications ${ }^{4}$ ), but heritability studies have suggested that genetic factors are also involved in the control of cardiac repolarization at the population level. ${ }^{5-8}$ In each individual, the activity and expression levels of cardiac ion channels establish a subtle equilibrium between depolarizing and repolarizing currents determining the action potential duration of ventricular cardiomyocytes. Mutations identified in ion channel encoding genes are responsible for familial ventricular arrhythmia disorders with high risk of sudden death, such as the short and long QT syndromes 
Table 1 SNPs in cardiac ion channel genes associated with QTC length in healthy Caucasian populations

\begin{tabular}{|c|c|c|c|c|c|c|c|c|}
\hline Gene & SNP region & Coding & $\begin{array}{c}\text { SNP } \\
\text { database }\end{array}$ & Alleles & $\begin{array}{l}\text { CDNA position } \\
\text { nomenclature }\end{array}$ & Genome position $^{\mathrm{a}}$ & $\begin{array}{l}\text { Initial association } \\
\text { studies }\end{array}$ & Association results \\
\hline KCNQ1 & Intron 1 & NC & rs757092 & $A>G$ & - & Chr11:2455754 & Pfeufer et al, 2005 & $\mathrm{G}>$ longest $\mathrm{QT} \mathrm{c}^{\mathrm{b}}$ \\
\hline \multirow[t]{2}{*}{ KCNH2 } & Exon 11 & K897T & rs1805123 & $A>C$ & 2690 & Chr7:150276467 & \multirow{2}{*}{$\begin{array}{l}\text { Bezzina et al, } 2003 \\
\text { Pfeufer et al, } 2005 \\
\text { Gouas et al, } 2005 \\
\text { Pfeufer et al, } 2005\end{array}$} & C > shortest QTC \\
\hline & 3'UTR & & rs3815459 & $G>A$ & - & Chr7:150275327 & & $\mathrm{A}>$ longest $\mathrm{QTC} \mathrm{c}^{\mathrm{b}}$ \\
\hline \multirow[t]{3}{*}{ SCN5A } & $\begin{array}{l}\text { Intron } 9 \\
\text { Exon } 12\end{array}$ & $\begin{array}{l}\text { NC } \\
\text { H558R }\end{array}$ & rs1805124 & $\begin{array}{l}C>A \\
A>G\end{array}$ & $\begin{array}{c}\text { IVS9-3 (1141-3) } \\
1673\end{array}$ & Chr3:38620424 & \multirow[t]{2}{*}{$\begin{array}{l}\text { Gouas et al, } 2005 \\
\text { Aydin et al, } 2005 \\
\text { Gouas et al, } 2005\end{array}$} & $\begin{array}{l}\text { A > longest QTc } \\
\mathrm{G}>\text { longest QTc }\end{array}$ \\
\hline & Intron 24 & NC & & $G>A$ & IVS24+116 & - & & $A>$ longest $Q T c^{d}$ \\
\hline & Exon 28 & D1819D & rs1805126 & $C>T$ & 5457 & Chr3:38567410 & Gouas et al, 2005 & $\mathrm{~T}>$ shortest QTC \\
\hline KCNE1 & $\begin{array}{l}\text { Intron } 2 \\
\text { Intron } 2 \\
\text { Exon } 3\end{array}$ & $\begin{array}{l}\text { NC } \\
\text { NC } \\
\text { G38S }\end{array}$ & $\begin{array}{l}\text { rs2236609 } \\
\text { rs1805127 }\end{array}$ & $\begin{array}{l}G>A \\
C>T \\
G>A\end{array}$ & $\begin{array}{l}\text { IVS2-128 } \\
\text { IVS2-129 } \\
112\end{array}$ & $\begin{array}{l}\text { chr21:34743980 } \\
\text { chr21:34743981 } \\
\text { chr21:34743691 }\end{array}$ & $\begin{array}{l}\text { Aydin et al, } 2005 \\
\text { present study } \\
\text { Friendler et al, } \\
2005 \\
\text { Gouas et al, } 2005 \\
\text { Akyol et al, } 2007\end{array}$ & $\begin{array}{l}\mathrm{G}>\text { longest } \mathrm{QTC} \mathrm{c}^{\mathrm{c}} \\
\text { no association } \\
\mathrm{A}>\text { longest } \mathrm{QTC} \\
\text { no association }\end{array}$ \\
\hline KCNE2 & Exon 2 & T8A & rs2234916 & $A>G$ & 22 & chr21:34664669 & Aydin et al, 2005 & $\mathrm{G}>$ shortest $\mathrm{QTC} \mathrm{C}^{\mathrm{C}}$ \\
\hline
\end{tabular}

${ }^{\mathrm{a}} \mathrm{db}$ SNP build 126.

$\mathrm{NC}$, noncoding.

All these SNPs have been studied in the D.E.S.I.R population (Gouas et al, 2005 or present study).

${ }^{\mathrm{b}}$ Associations confirmed in the present study.

cAssociations not confirmed.

${ }^{\mathrm{d}}$ Association of the allele A with the shortest QTc was found in this study.

characterized by shortened or prolonged QT intervals, respectively. ${ }^{9-12}$

Several groups have undertaken genetic association studies to identify QTc-associated polymorphisms in ion channel encoding genes in healthy subjects. ${ }^{13-18}$ Our group and others showed that the T897 allele (K897 T, rs1805123) in KCNH2 (LQT2), encoding the $\alpha$-subunit of the voltage-gated $\mathrm{I}_{\mathrm{Kr}}$ channel, and the R558 allele (H558R, rs1805124) in SCN5A (LQT3), encoding the cardiac voltage-gated sodium channel $\mathrm{Na}_{\mathrm{v}} 1.5$, have a shortening $^{14,16,18}$ and a prolonging ${ }^{15,16}$ influence on the QTC interval, respectively, in independent populations of French and German origin. Five other polymorphisms, SCN5A IVS24+116 G>A, KCNE1 IVS2-128 G>A, KCNE2 rs2234916 (T8A), KCNQ1 rs757092, KCNH2 rs3815459, have also been associated with QTc length in German populations $^{15,18}$ (Table 1), but these associations have never been confirmed in a population of different origin. We thus studied these five polymorphisms in our French D.E.S.I.R (Data from an Epidemiological Study on the Insulin Resistance syndrome) population.

\section{Materials and methods Study population}

Participants in the study Data from an Epidemiological Study on the Insulin Resistance syndrome (D.E.S.I.R) gave informed consent to the clinical and genetic study, which was approved by an ethics committee. The investigations conform to the principles outlined in the Declaration of Helsinki. Briefly, 3478 subjects underwent a 12-lead resting ECG at 3 years intervals (D0 and D3). QT intervals were measured in lead II (ms) by Cardionics ${ }^{\circledR}$ software and corrected for heart rate by use of the Fridericia formula (QTc). A total of 1470 subjects were excluded on the basis of a known or detected cardiac pathology, diabetes, treated or untreated high blood pressure, medication known to prolong the QT interval, and a difference $\geqslant 30 \mathrm{~ms}$ in QTc between D0 and D3. The features (mean age, mean heart rate, mean QTc interval and QTc range) of the 200 selected subjects (100 men and 100 women) with the shortest ageadjusted mean QTc, and of the 200 selected subjects (100 men and 100 women) with the longest age-adjusted mean QTc intervals, from the 2008 healthy D.E.S.I.R subpopulation, have been described previously. ${ }^{16}$

\section{Polymorphism typing}

Sample genotypes were determined using PCR and sequencing for the KCNH2 rs3815459 G>A, KCNE1 IVS2-128 $\mathrm{G}>\mathrm{A}, \quad$ KCNE1 rs2236609 (IVS2-129 C>T), KCNE1 rs1805127 (G38S) and KCNE2 rs2234916 (T8A) polymorphisms, and fluorescence resonance energy transfer (F.R.E.T) and probe melting curves for the KCNQ1 rs757092 A>G, 
SCN5A IVS24+116 G>A, SCN5A IVS9-3 C $>$ A and rs1805126, KCNH2 rs1805123 (K897 T), as described previously. ${ }^{16}$ The sequencing reactions were run on an ABI 3100 sequencer (Applied Biosystems, Foster City, USA) and genotypes analyzed using SEQSCAPE ${ }^{\mathrm{TM}}$ version 2.5 software (Applied Biosystems). Primers used to generate PCR products and hybridization probes used for F.R.E.T are given in Supplementary Table 1.

\section{Statistical analysis}

Deviation from the Hardy-Weinberg equilibrium was tested by $\chi^{2}$ analysis with $1 \mathrm{df}$, separately, in the shortest and longest QTc groups. Allele frequencies were estimated from genotype frequencies by gene counting. The study compared the longest and the shortest QTc groups. The odds ratio (OR) (95\% CI) for longest QTc associated with one copy of the minor allele was estimated from logistic regression analysis, adjusted for age and sex. Homogeneity of the association according to gender was systematically tested and as there was no heterogeneity for any of the polymorphisms, men and women were pooled for analysis. Estimation of the pairwise linkage disequilibrium (LD) coefficients between polymorphisms was expressed in terms of $\mathrm{D}^{\prime}=\mathrm{D} / \mathrm{Dmax}$ or $-\mathrm{D} / \mathrm{Dmin} .^{19}$ To estimate Haplotype frequencies, we combined the polymorphisms studied here with the polymorphisms previously genotyped, ${ }^{16}$ when they were not in almost complete concordance and had a frequency $>0.02$. In KCNQ1, polymorphisms located upstream exon 15 were in weak LD, generating multiple rare haplotypes. ${ }^{16}$ Thus, haplotype study for KCNQ1 polymorphisms combining the two SNPs in intron 1 and the SNPs located upstream exon 15 was not possible. Haplotype analyses were performed by use of a maximum likelihood model implemented in the THESIAS program (http://genecanvas.ecgene.net). ${ }^{20}$ A $P$-value $<0.05$ was considered statistically significant. All computations were carried out with SAS software (SAS Institute, Cary, NC, USA).

\section{Results \\ Association analysis of individual SNPs}

Five SNPs were genotyped in the 200 subjects with the shortest QTc and the 200 subjects with the longest QTc from a population of 2008 healthy subjects: KCNQ1 rs757092, KCNH2 rs3815459, SCN5A IVS24+116 G>A, KCNE1 IVS2-128 G>A, and KCNE2 rs2234916 (T8A). Genotype frequencies did not deviate from the HardyWeinberg equilibrium, in either group, for any of the studied SNPs.

The minor allele G of KCNQ1 rs757092 and A of KCNH2 rs3815459 were significantly more frequent in the longest than in the shortest QTc group (0.397 vs 0.312 for rs757092, 0.240 vs 0.172 for rs3815459). The OR (95\% CI) for the longest QTc associated with carrying the minor allele was $1.48(1.10-2.01), P=0.011$ for rs757092 and 1.48 (1.05-2.10), $P=0.026$ for rs3815459, respectively (Table 2 ).

Conversely, the minor allele A of SCN5A IVS24+116 was significantly more frequent in the shortest than in the longest QTc group (0.162 vs 0.104$)$, with OR (95\% CI) for the longest QTc associated with carrying the minor allele of 0.61 (0.40-0.92), $P=0.018$ (Table 2).

KCNE1 IVS2-128 G>A and KCNE2 rs2234916 A>G variations were rare in our population (0.019 and 0.005, respectively) and there was no significant association between QTc length and these polymorphisms. A more common KCNE1 polymorphism, 5' adjacent to IVS2-128 $\mathrm{G}>\mathrm{A}$, named IVS2-129 C>T (rs2236609), was also genotyped. Allele frequency did not differ significantly between the shortest and longest QTc groups (0.427 vs 0.421, respectively) (Table 2 ).

\section{Haplotype analysis}

Haplotypes were constructed for rs1805123 A>C (K897T) and rs3815459 G>A within KCNH2, IVS9-3 C $>$ A, IVS24 $+116 \mathrm{G}>\mathrm{A}$ and rs1805126 (D1819D) within SCN5A, and rs2236609 and rs1805127 (G38S) within KCNE1.

KCNH2 rs1805123 (K897T) and rs3815459 were in moderate LD $\left(\mathrm{D}^{\prime}=-0.60\right)$. Haplotype analysis confirmed the shortened QTc-associated effect observed for rs1805123 and the prolonged QTc-associated effect observed for rs3815459 and showed that their respective effects were independent of each other (data not shown, test for interaction between the two alleles $P=0.76$ ).

SCN5A IVS24 + $116 \mathrm{G}>\mathrm{A}$ was in tight LD with rs1805126 (D1819D) $\left(\mathrm{D}^{\prime}=+0.97\right)$ and in moderate LD with IVS9-3 $\mathrm{C}>\mathrm{A}\left(\mathrm{D}^{\prime}=-0.63\right)$. The minor allele IVS24 $+116 / \mathrm{A}$ belongs to a single haplotype combining IVS9-3/C and 5457/T. Thus, as no haplotype effect had previously been detected between $1141-3$ and $5457,{ }^{16}$ no additional information on haplotypes was provided by IVS24 +116 .

KCNE1 rs2236609 and rs1805127 were in moderate LD $\left(\mathrm{D}^{\prime}=+0.61\right)$. Haplotype analysis (Table 3) showed that haplotype combining alleles rs2236609 C and rs1805127 A (H3) was more frequent in the group with the longest QTc intervals, with OR $(95 \%$ CI) for the longest QTc associated with carrying one copy of haplotype (H3) of 1.97 (1.11-3.48), $P=0.020$, with reference to the most frequent haplotype $(\mathrm{H} 1)$.

\section{Discussion}

Twin- or family-based genome-wide linkage analyses have identified three QTc duration, influencing genomic loci on chromosomes 11p (LQT1), ${ }^{7} 4 \mathrm{q}$ (LQT4) ${ }^{7}$ and 3p. ${ }^{7,21}$ Polymorphic variants that may influence QTc duration in healthy populations of Caucasian origin have been assessed in genes encoding ion channel subunits. Some minor alleles of these polymorphisms were described as 
Table 2 Genotype counts and allele frequency of the polymorphisms in the shortest and longest QTc groups and odds ratio $(95 \% \mathrm{Cl})$ for longest QTc associated with one copy of minor allele (adjusted for age and sex)

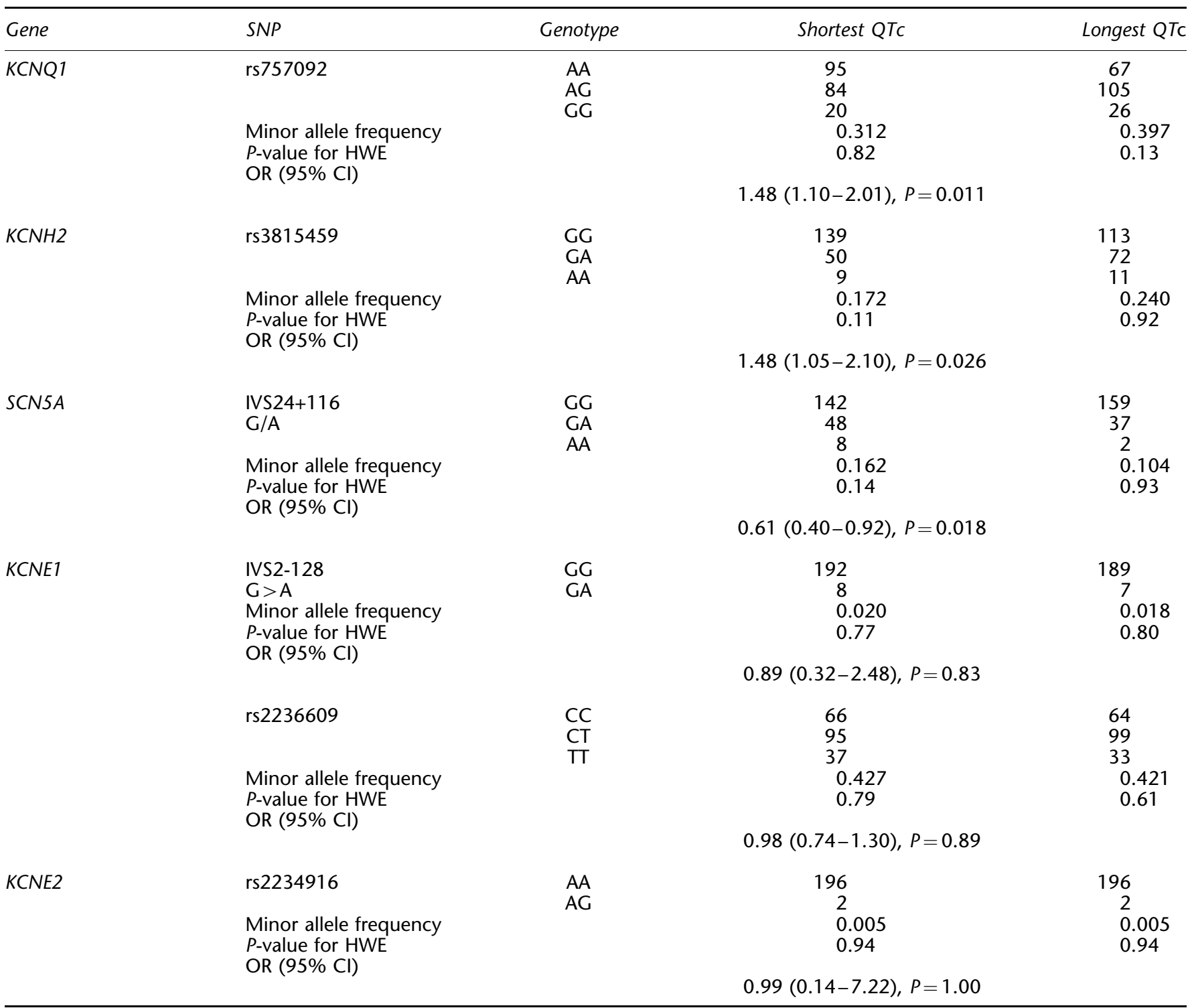

Table 3 The estimated KCNE1 haplotype frequencies in the group with the shortest and the group with the longest QTC intervals and odds ratios for the haplotypes

\begin{tabular}{|c|c|c|c|c|c|}
\hline & $r s 2236609$ & rs1805127 (G38S) & Shortest QTC $(N=392$ alleles $)$ & Longest QTC $(N=396$ alleles $)$ & OR $(95 \% C l)^{\mathrm{a}}$ \\
\hline $\mathrm{H} 1$ & $\mathrm{C}$ & G & 0.517 & 0.468 & ref \\
\hline $\mathrm{H} 2$ & $\bar{T}$ & G & 0.119 & 0.152 & $1.30(0.86-1.97), P=0.21$ \\
\hline $\mathrm{H} 3$ & C & A & 0.056 & 0.111 & $1.97(1.11-3.48), P=0.02$ \\
\hline $\mathrm{H} 4$ & $\mathrm{~T}$ & A & 0.308 & 0.269 & $0.97(0.69-1.37), P=0.87$ \\
\hline
\end{tabular}

$P=0.081$ for the global association.

${ }^{a}$ Odds ratios with $95 \%$ confidence intervals $(95 \% \mathrm{Cl})$ were calculated with reference to the most common haplotype $\mathrm{H} 1$.

either associated with a shortening or a lengthening of QTc. ${ }^{14,16,18,21}$ However, few of these results have been replicated in independent populations. In this study, we tested SNPs KCNQ1 rs757092 A>G, KCNH2 rs3815459 $\mathrm{G}>\mathrm{A}, \quad S C N 5 A$ IVS24 + $116 \mathrm{G}>\mathrm{A}$, KCNE1 IVS2-128 G>A and KCNE2 rs2234916 A $>\mathrm{G}$ for association with QTc 
length in our population. Correction for multiple testing was not required as a small number of polymorphisms was studied, and we intended to verify the previously observed associations.

We found that the alleles KCNQ1 rs757092 G and KCNH2 rs3815459 A were more frequent in the group of subjects with the longest QTc, which confirm the initial association described in a German population. ${ }^{18}$

We found that allele SCN5A IVS $24+116$ A was more frequent in the group of subjects with the shortest QTc. In the initial study, Aydin et $a l^{15}$ reported an association between this polymorphism and QTc length in a normal German population of twins and showed that subjects with SCN5A IVS24+116 A/A genotype had longer QTc than subjects with SCN5A IVS24+116 G/G. Additional studies in large series are needed to elucidate whether or not this polymorphism is associated with QTc length.

The alleles KCNE1 IVS2-128 A and KCNE2 rs2234916 G were very rare and the allele frequency was not significantly different between the two groups for any of them. Nevertheless, haplotype analysis of two common KCNE1 SNPs (rs2236609 and rs1805127 (G38S)) showed a twofold increased risk of QTc lengthening when carrying the haplotype combining alleles rs2236609 C and rs1805127 A. In our previous study, there was no evidence that SNP rs1805127 was associated with QTc length, ${ }^{16}$ as recently reported in a large population sample, ${ }^{17}$ whereas Friedlander $e a^{22}$ reported its association with the QTc interval in men. In this latter study, variance-component linkage analysis revealed weak evidence of linkage of KCNE1 SNPs with QTc interval, whereas family-based association analysis demonstrated a significant association between SNP rs1805127 and QTc interval, suggesting that SNP rs1805127 could have a modest effect and act as a susceptibility locus on the QTc trait. This modest effect could be detected only by haplotype analyses in our population. Further association studies with KCNE1 markers would enable the role of KCNE1 on QTc length to be settled.

In SCN5A, IVS24+116 in intron 24 and rs1805126 (D1819D) in exon 28 were in nearly complete positive LD in our population, contributing to the same trend of association of their alleles with the shortest QTc. ${ }^{16}$ Both these polymorphisms were in weak LD with IVS9-3 C $>$ A SNP, which was associated with prolonged QTc in our previous study. ${ }^{16}$ In KCNH2, rs1805123 (K897T) and rs3815459 were in moderate negative LD and showed independent and opposite effects on QTc length. High-resolution LD mapping studies of these genes should enable the identification of haplotype blocks and relevant tagging SNPs for genetic association studies. Further investigations are needed to identify the functional variants that could explain the opposite and independent effects observed for some SNPs lying in the same gene.
In summary, our study replicated the associations reported for SNPs rs757092 in KCNQ1 and rs3815459 in $K C N H 2$, reported association of allele A of IVS24+116 $\mathrm{G}>\mathrm{A}$ in SCN5A with the shortest QTc and failed to replicate the associations of IVS2-128 G>A in KCNE1 and rs2234916 (T8A) in KCNE2. It is possible that these conflicting results could be resolved with further investigations using larger sample sizes.

\section{Acknowledgements}

This work was supported by the INSERM, Fondation de France, and Fondation Leducq. The design of hybridization probes for the Fluorescence Resonance Energy Transfer assays was from TIB MOLBIOL (Germany).

\section{References}

1 Bazett H: An analysis of the time relations of electrocardiograms. Heart 1920; 7: 353-367.

2 Reardon M, Malik M: QT interval change with age in an overtly healthy older population. Clin Cardiol 1996; 19: 949-952.

3 Yang H, Elko P, LeCarpentier GL et al: Sex differences in the rate of cardiac repolarization. J Electrocardiol 1994; 27 (Suppl): 72-73.

4 Kaab S, Hinterseer M, Nabauer M, Steinbeck G: Sotalol testing unmasks altered repolarization in patients with suspected acquired long-QT-syndrome - a case-control pilot study using i.v. sotalol. Eur Heart J 2003; 24: 649-657.

5 Russell MW, Law I, Sholinsky P, Fabsitz RR: Heritability of ECG measurements in adult male twins. J Electrocardiol 1998; 30 (Suppl): 64-68.

6 Carter N, Snieder H, Jeffery S et al: QT interval in twins. J Hum Hypertens 2000; 14: 389-390.

7 Busjahn A, Knoblausch H, Faulhaber H-D et al: QT interval is linked to 2 long-QT syndrome loci in normal subjects. Circulation 1999; 99: 3161-3164.

8 Friedlander Y, Lapidos T, Sinnreich R, Kark JD: Genetic and environmental sources of QT interval variability in Israeli families: the kibbutz settlements family study. Clin Genet 1999; 56: $200-209$.

9 Bellocq C, van Ginneken AC, Bezzina CR et al: Mutation in the KCNQ1 gene leading to the short QT-interval syndrome. Circulation 2004; 109: 2394-2397.

10 Wolpert C, Schimpf R, Giustetto C et al: Further insights into the effect of quinidine in short QT syndrome caused by a mutation in HERG. J Cardiovasc Electrophysiol 2005; 16: 54-58.

11 Priori SG, Pandit SV, Rivolta I et al: A novel form of short QT syndrome (SQT3) is caused by a mutation in the KCNJ2 gene. Circ Res 2005; 96: 800-807.

12 Splawski I, Shen J, Timothy K et al: Spectrum of mutations in long QT syndrome genes KVLQT1, HERG, SCN5A, KCNE1, and KCNE2. Circulation 2000; 102: 1178-1185.

13 Pietila E, Fodstad H, Niskasaari E et al: Association between HERG K897 T polymorphism and QT interval in middle- aged Finnish women. J Am Coll Cardiol 2002; 40: 511-514.

14 Bezzina CR, Verkerk AO, Busjahn A et al: A common polymorphism in KCNH2 (HERG) hastens cardiac repolarization. Cardiovasc Res 2003; 59: 27-36.

15 Aydin A, Bahring S, Dahm S et al: Single nucleotide polymorphism map of five long-QT genes. J Mol Med 2005; 83: $159-165$.

16 Gouas L, Nicaud V, Berthet $\mathrm{M}$ et al: Association of KCNQ1, KCNE1, KCNH2 and SCN5A polymorphisms with QTc interval length in a healthy population. Eur J Hum Genet 2005; 13: $1213-1222$ 
17 Akyol M, Jalilzadeh S, Sinner MF et al: The common nonsynonymous variant G38S of the KCNE1-(minK)-gene is not associated to QT interval in Central European Caucasians: results from the KORA study. Eur Heart J 2007; 28: 305-309.

18 Pfeufer A, Jalilzadeh S, Perz S et al: Common variants in myocardial ion channel genes modify the QT interval in the general population: results from the KORA study. Circ Res 2005; 96: 693-701.

19 Tiret L, Amouyel P, Rakotovao R, Cambien F, Ducimetiere P: Testing for association between disease and linked marker loci: a log- linear-model analysis. Am J Hum Genet 1991; 48: 926-934.
20 Tregouet DA, Escolano S, Tiret L, Mallet A, Golmard JL: A new algorithm for haplotype-based association analysis: the StochasticEM algorithm. Ann Hum Genet 2004; 68: 165-177.

21 Newton-Cheh C, Larson MG, Corey DC et al: QT interval is a heritable quantitative trait with evidence of linkage to chromosome 3 in a genome-wide linkage analysis: The Framingham Heart Study. Heart Rhythm 2005; 2: 277-284.

22 Friedlander Y, Vatta M, Sotoodehnia N et al: Possible association of the human KCNE1 (minK) gene and QT interval in healthy subjects: evidence from association and linkage analyses in Israeli families. Ann Hum Genet 2005; 69: 645-656.

Supplementary Information accompanies the paper on European Journal of Human Genetics website (http://www.nature.com/ejhg) 